\title{
The Effects of UPcomplish on Office Workers' Sedentary Behaviour, Quality of Life and Psychosocial Determinants: A Stepped-Wedge Design
}

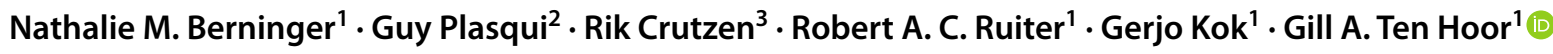

Accepted: 13 January 2022 / Published online: 31 January 2022

(c) The Author(s) 2022

\begin{abstract}
Background Sedentary behaviour (SB) affects cardiometabolic health and quality of life (QoL). We examine the effects of UPcomplish, a 12-week data-driven intervention, on SB, QoL and psychosocial determinants among office workers.

Methods Participants were recruited via judgement sampling. Five groups starting with time-lags of 7 weeks $(n=142,96$ females) received 14 feedback messages (FBMs) which were tailored to SB patterns, goals and hurdles. Participants received questionnaires at the beginning, middle and end of the intervention and wore an accelerometer measuring SB, operationalized as proportions (compositional data approach, CoDA) and summed squared sitting bouts (SSSB). We used linear mixed-effects models with random intercepts for weeks (between-subjects) and individuals (within-subjects).

Results UPcomplish did not reduce SB. Within-subjects compared to baseline, FBM \#3 ( $\beta$ CoDA $=0.24, p<.001,95 \%$ CI $[0.15,0.33] ; \beta S S S B=20.83, p<.001,95 \%$ CI [13.90, 27.28]) and \#4 ( $\beta \mathrm{CoDA}=0.20, p<.001,95 \%$ CI $[0.11,0.29]$; $\beta S S S B=24.80, p<.001,95 \%$ CI $[15.84,33.76])$ increased SB. QoL was unaffected. Perceived susceptibility was lower after FBMs \#6 to \#8 ( $\beta$ between $=-0.66, p=.04,95 \%$ CI $[-1.03,-0.30]$; $\beta$ within $=-0.75, p=.02,95 \%$ CI $[-1.18,-0.32]$ ). Within-subjects, intentions to sit less were higher after FBMs \#1 to \#5 (1.14, $p=.02,95 \%$ CI [0.61, 1.66]). Improvements in determinants and in SB were not associated, nor were improvements in SB and in QoL.

Conclusions Compared to VitaBit only, UPcomplish was not beneficial. Environmental restructuring might be superior, but detailed analyses of moderators of effectiveness are needed.
\end{abstract}

Keywords Sedentary behaviours · Intervention mapping · Quality of life · Vitality · Office workers · Compositional data approach

\section{Abbreviations}

SB Sedentary behaviours

QoL Quality of life

FBM Feedback message
MVPA Moderate-to-vigorous physical activity

IM Intervention mapping
Gill A. Ten Hoor

gill.tenhoor@maastrichtuniversity.nl

Nathalie M. Berninger

nathalie.berninger@maastrichtuniversity.nl

Guy Plasqui

g.plasqui@maastrichtuniversity.nl

Rik Crutzen

rik.crutzen@maastrichtuniversity.nl

Robert A. C. Ruiter

r.ruiter@maastrichtuniversity.nl

Gerjo Kok

g.kok@maastrichtuniversity.nl
1 Department of Work and Social Psychology, Maastricht University, P.O. Box 616, 6200 MD Maastricht, The Netherlands

2 Department of Human Biology and Movement Sciences, Nutrition and Translational Research in Metabolism, Maastricht University, P.O. Box 616, 6200 MD Maastricht, The Netherlands

3 Department of Health Promotion, CAPHRI, Maastricht University, P.O. Box 616, 6200 MD Maastricht, The Netherlands 


\section{Introduction}

Type 2 diabetes, cardiovascular disease $[1,2]$ and mental health problems [3] are potential consequences of sedentary behaviours (SB), which include sitting, lying or reclining behaviours (excl. sleeping) that exhibit low energy expenditures [4]. Except for amounts of more than $10 \mathrm{~h}$, not the sitting time per se seems to be detrimental, but a pattern with bouts of long, uninterrupted SB [5, 6]. Indeed, regular SB interruptions of standing and light activity with the same energy expenditure as single bouts of MVPA seem to be at least equally effective in reducing cardiometabolic risk [7].

The mechanisms of how SB affects physical and mental health are complex. During SB, the muscles of the lower limbs are static, which reduces blood flow, downregulates endothelial functions, and increases inflammation [1]. These aspects yield physical problems but also impact brain health and quality of life (QoL) [8-10]. For example, SB involves low muscle contractions suppressing the lipoprotein lipase in red muscle fibres [11]. Ineffective triglyceride metabolism and visceral fat increase insulin resistance and reduce binding of leptin in the hypothalamus and hippocampus, which is responsible for synaptic plasticity [8]. Moreover, cerebral blood flow and the release of neurotrophines are reduced during SB [9]. These mechanisms might impair cognitive functioning, vitality and thus performance [10]. Furthermore, prolonged SB increases the pressure on the intervertebral disks and weakens posterior lumbar structures, explaining its link to increased intensities of lower back pain $[12,13]$, and to neck and upper extremity musculoskeletal symptoms [14]. Lastly, despite a lack of clarity about the mechanisms, SB has been linked to stress and mental health problems $[15,16]$.

Since modernization yielded a higher prevalence of office work including about $60 \%$ of SB, interventions effective in reducing SB among office workers are needed [17]. Interventions that have been effective seem to include either environmental changes such as standing desks or personal coaches in addition to persuasion techniques [18-20]. Using technology to communicate tailored feedback and advice might be a more cost-efficient way than regular personal coaching [21-23]. Yet, purely computertailored SB interventions having shown reductions in workplace SB did not find these effects when combining working and leisure time SB [24-26]. Therefore, a personal coach providing tailored but automated feedback might be the optimal mixture of a low-cost yet personal intervention.

We applied the intervention mapping (IM) protocol to systematically develop a data-driven SB intervention aimed at a reduction of SB among office workers: UPcomplish [28, 29]. Workplace physical activity interventions that have been developed with IM have yielded promising effects [30-32]. The development of UPcomplish using evidence from the literature and from theories (e.g. reasoned action approach [33]) is described elsewhere [27]. Shortly, the problem of SB was refined, and behavioural outcomes, and performance objectives (i.e. sub-behaviours), were formulated. Important and changeable psychosocial determinants (e.g. attitude, perceived behavioural control, PBC) were linked to the performance objectives. We selected evidence-based behaviour change methods and translated them into practical applications by the help of parameters for use and by considering the change objectives (i.e. change needed in the determinants to realize the performance objectives) $[34,35]$. For example, the method "consciousness raising" can help to change "attitude", which will likelier yield a decision to reduce SB if the rise of awareness (i.e. of negative consequences of $\mathrm{SB}$ ), is rapidly followed by an increase in self-efficacy [29, 35]. Pre-tests and a pilot test facilitated further refinement and the automation of the 14 feedback messages (FBMs) [27]. The main component was "UPcomplish" consisting of 14 FBMs, which automatically tailored to participants' SB patterns, and were send by a coach. The FBMs tackled self-efficacy, attitude, perceived social support (PSS), perceived susceptibility (PS), and normative beliefs through implementation intentions, monitoring, tailored feedback, and motivational support [36]. The second component, VitaBit, served as monitoring toolkit providing information, at which point in time participants were sitting, standing, or moving [37].

The primary objective of this study was to investigate the effects of UPcomplish on objectively measured SB, self-reported QoL (i.e. perceived performance, stress, pain, emotional well-being, EWB, vitality), and psychosocial determinants (i.e. attitude, PSS, PBC, PS, intention). Between and within subjects, we expected UPcomplish to reduce the daily proportion of SB and prolonged sitting when compared to monitoring only phases, i.e. baseline periods. Furthermore, we expected improvements in QoL and in the psychosocial determinants. The secondary objective was to explore correlates between the variables being assessed. We expected that the psychosocial determinants would be correlated with SB, and that SB would be correlated with QoL. We chose a stepped-wedge design (Fig. 1) above a parallel randomized control trial to reduce the burden for participants in a potential waiting control group (e.g. compliance), to increase statistical power (i.e. groups act as both control and intervention group; continuously measured SB) [38], and to gather seasonal spread data. 


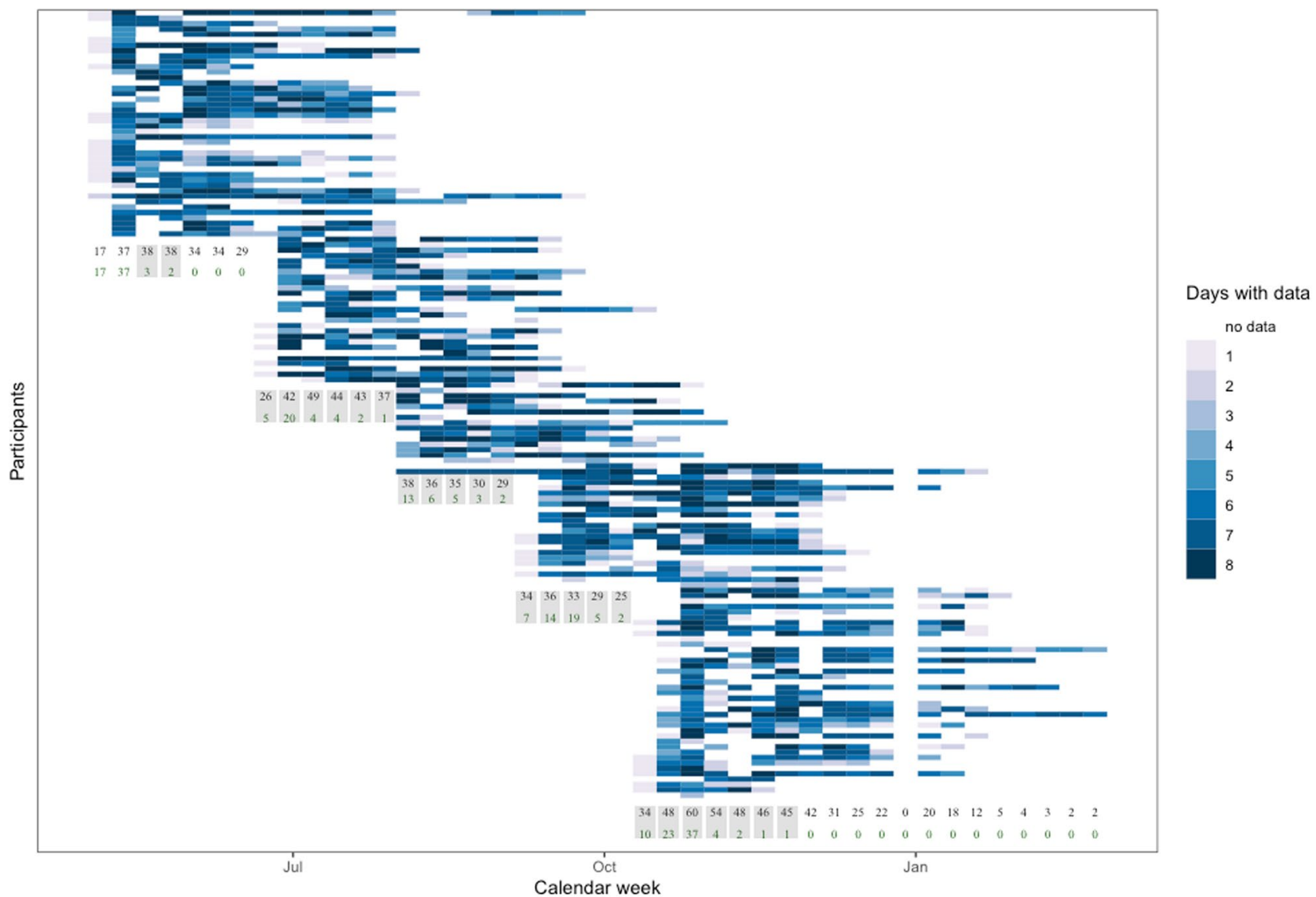

Fig. 1 Flowchart of the stepped-wedge trial of the UPcomplish intervention. The black numbers indicate per week, how many participants provided data; the green numbers how many participants were in their

\section{Methods}

This study was pre-registered: NL7503 (https://www. trialregister.nl/trial/7503). The intervention protocol can be found in [27]. The cleaned raw data and additional material are fully disclosed in the supplementary materials (https://osf.io/ qzp9m/?view_only=30ada8d6fc0e4ac19a1610b8901f9f96). We adhere to the Consolidated Standards of Reporting Trials (CONSORT) checklist of information to include when reporting a stepped wedge cluster randomized trial [39].

\section{Study Design and Sample}

Five intervention groups (Fig. 1) of maximum 40 participants started with time lags of about 7 weeks (exact duration depended on holidays and availabilities). Participants were eligible to take part if they were able to stand and walk, and willing to download the VitaBit app on their smartphones (at least Android 4.3 or iOS 8.1). Furthermore, only people who defined themselves as office workers and who understood the baseline phase during the concerning week. Weeks with both baseline and intervention data, which are relevant for the between-subjects' comparisons, are marked with grey cuboids

German language could participate. If any of the inclusion criteria were not met, participants were excluded.

All groups participated in a kick-off (incl. generic information on SB and the health consequences, which in itself has not been linked to changes in SB before [40]). To create a personal atmosphere and considering time constraints but still be efficient, the kick-offs were held for different sub-groups (i.e. companies) with a maximum of 15 and a minimum of 5 participants. The sub-groups were continuously recruited starting in one of the upcoming kick-offs that they would be available. If many participants per group dropped out, sub-groups were merged to still allow for group activities (e.g. challenges, group report). A baseline week after the kick-off session served as the control condition, where participants wore the VitaBit without receiving any tailored FBMs. After the baseline week, everyone received the intervention consisting of 14 FBMs. Each FBM targeted specific psychosocial determinants and change objectives, but were tailored to individual behaviours and goals (e.g. a suggestion of a goal targeted PBC but contained different goals per individual). 
All individuals were compared to their baseline week (within-subjects' comparisons). Some of the calendar weeks included participants being at their baseline and participants having already received the intervention (between-subjects' comparisons). To test whether a certain dose of the intervention might be necessary to be effective, the reception of different amounts of FBMs being sent were analysed in separate regression models. For example, in calendar week 27, 42 participants had worn the VitaBit device. Among these, 20 participants had still been in their baseline week, and 12 had just received FBM \#5. When analysing the betweensubjects' effect of FBM \#5, these 12 participants were compared to the 20 participants being in their baseline week. To increase statistical power, the FBMs were aggregated when analysing the effects on psychosocial determinants and QoL, since these were assessed via surveys at three times: baseline (T0), week 6 (T1) and at the end of the intervention (T2).

For the time of the evaluation (May 2019-January 2020), we had 200 VitaBit sensors to our disposal. With an anticipated drop-out rate of $20 \%$ and five intervention groups (32 participants per group after drop-out and the middle group providing data for both baseline and intervention), we expected to end with a sample size of 192, which would reveal sufficient power according to our sample size planning [27].

Participants could refuse participation at all times, without giving a reason. Yet, most participants that dropped out gave a reason (e.g. technical problems, time constraints). This study and its consent procedure was approved by the Ethics Review Committee of the Faculty of Psychology and Neuroscience, Maastricht University, the Netherlands [ERCPN-188_11_02_2018]. The trial was pre-registered under: NL7503.

\section{Procedure}

\section{Recruitment}

The UPcomplish coach (psychologist employed by VitaBit software) was trained by the intervention developers. We contacted as many German companies as resources allowed (selected via judgement sampling; number not noted) via contact persons, personal conversations, emails, and phone calls and distributed the flyers. These included information on what would be expected from participants (e.g. downloading the app), and how much time participation would require (i.e. $1 \mathrm{~h}$ kick-off, $14 \times 2 \mathrm{~min}$ feedback, and $3 \times 20 \mathrm{~min}$ surveys). Additionally, it included information about inclusion criteria (e.g. being a desk worker, able to walk) and about the benefits one could expect from participation (e.g. vitality through a reduction of SB). As soon as the management of the companies agreed, a date for the kick-off was arranged. The participants were invited via email and received an instruction on the creation of a VitaBit account, the information sheet and the informed consent.

\section{Kick-Off and Measurement Time-Points}

For the kick-offs, the coach visited the participants in their companies. The duration depended on the size of the concerning sub-group and the number of questions (35 to $60 \mathrm{~min}$ ). The introduction including an estimation of participants' daily sitting times (on workdays and on days off) was followed by an explanation about the consequences of SB and by information about how UPcomplish could help them to reduce SB. Afterwards, participants were told to choose a realistic but challenging goal (e.g. sitting for a max. of $8 \mathrm{~h}$ per day), which would be adapted after the baseline week if necessary. These goals served as orientation for the participants and as basis to give first tailored advice. ${ }^{1}$ The coach explained the functionalities of the VitaBit toolkit and clarified questions. Participants were informed that they would receive an individual and a group report (i.e. at company level) and a $50 €$ VitaBit voucher as compensation for their participation. Furthermore, they received information on the purpose of study, detailed information on the participation procedure, data management, and potential benefits, before written informed consent was obtained. The VitaBit devices were distributed and connected via Bluetooth with the smartphone app. Participants who were not able to make it to the kick-off received an email with the information and hand-outs. At the end of the kick-off, the participants started wearing the VitaBit device. The week after the kick-off served as baseline and involved the first survey on QoL and on determinants. Afterwards, participants received the intervention including the second survey in week 6 and the last survey after the intervention. The 4 weeks after the intervention served as follow-up measurement and participants received their compensation, before the devices were collected.

\section{Intervention}

The protocol of the intervention and the link between the FBMs and the psychosocial determinants are described elsewhere [27]. For each FBM, the authorized coach downloaded the raw data (pseudonymized IDs and SB) from the

\footnotetext{
1 Although the intervention and the advice focused on the reduction of SB (e.g. drink more to sit less), participants could also set a physical activity goal (e.g. being active for at least $1 \mathrm{~h}$ per day), which enabled a provision of choice. Participants with physical activity goals received similar feedback on SB patterns (e.g. your sitting bouts are the longest on Tuesday mornings) but different feedback on the achievement of goals (e.g. you reached your goal of moving at least $1 \mathrm{~h}$ on Wednesday).
} 
Table 1 Overview of the FBMs of the UPcomplish intervention

\begin{tabular}{lll}
\hline & & Delivery $^{\mathrm{a}}$ \\
\hline$\# 1$ & Goal adaption and sub-goals & Monday, week 2 \\
$\# 2$ & Feedback sitting pattern and first challenge & Thursday, week 2 \\
$\# 3$ & What are your hurdles to sit less? & Monday, week 3 \\
$\# 4$ & Tips how to overcome hurdles & Thursday, week 3 \\
$\# 5$ & Feedback on goal achievement & Monday, week 4 \\
$\# 6$ & Feedback sitting pattern and second challenge & Thursday, week 4 \\
$\# 7$ & Goal adaption and long-term goal & Monday, week 5 \\
$\# 8$ & Feedback on sitting pattern and on goal achievement & Thursday, week 5 \\
$\# 9$ & Feedback goal achievement and third challenge & Thursday, week 6 \\
$\# 10$ & Did your hurdles change? & Thursday, week 7 \\
$\# 12$ & Feedback goal achievement and last sub-goal & Thursday, week 8 \\
$\# 13$ & Feedback sitting pattern and fourth challenge & Thursday, week 9 \\
$\# 14$ & Competing colleagues & Thursday, week 10 \\
\hline
\end{tabular}

${ }^{\text {a }}$ Point in time if device is worn at all points in time server. The data were imported into $\mathrm{R}$ statistical software where the code cleaned and transformed them in such a way, that it provided the coach with tailored FBMs for all participants (either with the next FBM or with a reminder). The coach delivered the FBMs through the participants' preferred communication-channel (WhatsApp or email). Participants received two FBMs per week, which was reduced to one FBM per week as of week 6 (see Table 1 for an overview of the FBMs). The FBMs were not delivered, if a participant had dropped out, was on a holiday (if they indicated to pause for their holidays), or if not enough data were available (i.e. depending on the FBM less than 1 to 3 days à $6 \mathrm{~h}$ of data). If insufficient data were available on a feedback day, instead of receiving the next FBM, they received a reminder to synchronize their data or were asked if they still participated (maximum two reminders in a row). In case a participant received a reminder or did not receive any message, the concerning FBM was sent in the week after and the following FBMs were delayed also. FBMs \#13 (Competing colleagues) and \#14 (Tips how to keep new habits in the future) were not delayed and sent to all active participants in the last two weeks. Therefore, if participants missed two FBMs, they received FBMs \#1 to \#10, \#13 and \#14. Based on the baseline data and the goals from the kick-off meeting, the goals were adapted if necessary and broken down into graded sub-goals. In addition to feedback about goals, participants received tailored FBMs about their SB pattern (e.g. On Tuesday noons between 11:00 and 14:00[...], your sitting periods seem to be specifically long. Here is a tip [...]), questions about individual hurdles (e.g. What hinders you most when reducing your sitting behaviour? Is it habits, lack of time, $[\ldots]$ ?), and tailored tips to overcome personal hurdles. The FBMs also included challenges in biweekly circles. The last two weeks focused on sustaining new behaviours by the help of if-then-plans and finding a buddy. The second intervention component was the VitaBit app including tools to monitor SB, such as a "Vitality score" $(0=$ unhealthy SB pattern, $100=$ healthy SB pattern), the current amount of SB, and goal achievements.

\section{Measures}

\section{Behavioural Measurements}

Physical behaviour was continuously measured using accelerometery [41]. The VitaBit sensor $(3.9 \times 1.4 \times 0.85 \mathrm{~cm}$, $4.8 \mathrm{~g}$ ) was worn in trouser pockets or at the thigh (i.e. attached with a magnet). The battery life of the device is at least 30 days, and it shows sensitivity and specificity values of $85.7 \%$ and $91.2 \%$, respectively, for SB [37]. The device deploys a sampling rate of $33 \mathrm{~Hz}$ and an output data rate of $30 \mathrm{~s}$. Data are stored on the device for at least 30 days and can be synchronized with the VitaBit app via Bluetooth. Via Internet, the data are sent to a back-end server, where they are processed and stored (pseudonymized) in a time series database. An authorized coach can download them from the portal. The data cleaning procedure to retrieve the SB variables is described under the "Data Preparation" section.

The performance objectives (e.g., participants create a VitaBit account) were retrieved from behavioural observations. These will be analysed as potential moderators of effectiveness in a future article and are described in more detail elsewhere [27].

\section{Online Survey}

The survey was distributed at baseline (T0), after 6 weeks (T1), and after the intervention (T2). Sociodemographic and 
job-related variables were measured at $\mathrm{T} 0$, intervention characteristics (e.g., acceptability, understandability) at T2. Psychosocial determinants and QoL were measured at all three time points. We translated the Individual Work Performance Questionnaire into German using back-translation [42]. As indicators for reliability, we present Omegas $(\omega)$ if more than 2 items were used for a construct, and Pearson correlations $(r)$ if only two items were used $[43,44]$.

VitaBit obtained gender, age, education, height, weight, and job-related variables when participants created the account. They could choose between 8 educational degrees (e.g., Master's degree), between 29 job titles (e.g., sales, administrative), between 17 company industries (e.g., service, finance), and between different team sizes. At T0, they were asked about the usual number of workdays per week (from 1 to $7 ; 1$ item), employment status (full-time/parttime; 1 item) and job tasks (5 items). These included phone calls, computer work, desk work, having meetings, and travelling/visiting clients, e.g. "How much—on average per day (in \%)—do you estimate that you spend on the following tasks? Phone calls?" [45].

Task and contextual performance were assessed by subscales of the Individual Work Performance Questionnaire ( seldom $=0$ to always $=5$ ). Task performance ( 5 items; $\omega=0.72$ ) refers to the ability of performing the tasks being required for the job, operationalized as work quantity and quality or job skills, e.g. "During the last week, I was able to perform my work well with minimal time and effort". Contextual performance ( 9 items; $\omega=0.57$ ) refers to the organizational, social, or psychological requirements facilitating functioning at work, such as investing effort or cooperating, e.g. "I took on extra responsibilities." [46]. Stress perception was administered by the Perceived Stress Scale (10 items; e.g. "How often have you felt nervous and 'stressed'?"; $\omega=0.89$ ) [47, 48]. Bodily pain (2 items; e.g. "How much bodily pain have you had?"; $r=0.85$ ), EWB ( 5 items; e.g. "How much of the time have you been a happy person?"; $\omega=0.83$ ), and vitality (4 items; e.g. "How much of the time did you have a lot of energy?"; $\omega=0.86$ ) were assessed by subscales of the SF-36 [49].

We assessed the psychosocial determinants by questions about how much they agreed with certain statements. The items for attitudes (6 items; e.g. "[...] walking around at work is healthy"; $\omega=0.62$ ), PSS ( 2 items; e.g. " [...] walking around at work is encouraged by my colleagues"; $r=0.62$ ), PBC (4 items; e.g. "I am sure that I can [...] walk around at work, even though I feel bad, tired, tense or depressed"; $\omega=0.70$ ), and intention ( 2 items; e.g. "Are you planning to interrupt long sitting periods at work with [...] walking breaks?"; $r=0.43$ ) were based on former evaluation papers [45]. Additionally, we assessed PS, which refers to the belief to be at risk of getting a disease ( 2 items; e.g. "My daily sitting time is more compared to what is recommended."; $r=0.72)[50,51]$.

\section{Data Preparation}

Activity, survey data, and dates of received FBMs were merged using pseudonymized user identifiers. Since the three physical behaviour levels are multicollinear (e.g. more sitting results in less standing and walking), we applied a compositional data analysis approach (CoDA) to transform them into non-interdependent variables [52]. We transformed the proportions of the three physical behaviours in relation to the entire waking day (i.e. when the device was worn) into isometric log-ratios by adjusting for the proportions spent in the other two behaviours (i.e. $z 1_{\text {sitting }}=\sqrt{2 / 3} \ln ($ Sitting\% $/ \sqrt{\text { Standing\%xActivity\% }})$ [ [53]. To analyse the effects on prolonged sitting, we used the sum of the squared sitting bouts (SSSB) [27]. To weigh longer sitting bouts more than shorter bouts, daily sitting bouts are squared before being summed up ( SSSB $=\sum_{0}^{n}$ SitBout $\left._{i}^{2}\right)$. Afterwards, the data were cleaned to retain only those days that a participant collected enough data. Since there is always a trade-off between the retention of a high number of days and the retention of long days [54], we inspected the data by a plot: how many days would be retained for which daily wear time cut-off. Each stricter wear time cutoff resulted in fewer analysable days. The wear time cut-off of $8 \mathrm{~h}$ per day seemed to be a turning point (see Appendix A): each additional hour of required wearing time drastically reduced the number of available days. Therefore, only days with at least $8 \mathrm{~h}$ of VitaBit data were retained. Holidays were excluded from the analyses.

We created 14 variables with Boolean values representing whether the concerning FBM was already received at the concerning point in time, e.g. FBM_4_Received (TRUE/ FALSE/NA). These variables were FALSE, if a participant had not received any FBM (i.e., baseline week), and TRUE, if a participant had just received the concerning FBM (e.g. \#4). The variables were NA, if a participant had received more or less FBMs than the concerning FBM. The NAs were removed in the regression models to disentangle intervention effects from all other FBMs. Therefore, the reception of FBM \#4 (i.e. FBM_4_Received = TRUE) was compared against baseline (i.e. FBM_4_Received=FALSE). For each individual, the days were averaged by FBM, for example, all days after FBM \#4 but before \#5 were averaged. Outliers were excluded using the Mahalanobis distance method (generalized squared distance), which is used for multidimensional data and is defined as the distance of each point (row in the matrix) from a distribution, normalized by the standard deviation, and adjusted by the covariances of the variables [55]. 


\section{Data Analyses}

We used histograms and QQ plots to assess the distribution of the data. Non-normally distributed variables were reported as medians and interquartile ranges (IQRs), normally distributed variables as means and standard deviations (SDs), and categorical variables as absolute numbers and percentages.

To examine the between-subjects' effects of UPcomplish on $\mathrm{SB}, \mathrm{QoL}$ and psychosocial determinants, we used linear mixed-effects models with random intercepts for calendar week (which was dropped for QoL and the determinants, due to singularity). For comparability, all outcome variables were centred around the baseline sub-group means, and nonnormally distributed variables (i.e. SSSB) were transformed to a normal distribution using square roots. For assessing within-subjects' effects, the outcome variables (SB, QoL, and determinants) were centred around calendar week means (of baseline data), before deploying linear mixed-effects models with random intercepts for user identifier.

As a post hoc analysis, to analyse whether within-subjects' improvements (centred around calendar week means) in determinants, in SB, and in QoL were associated, we conducted pairwise Pearson correlations. Changes were calculated by subtracting the values at $\mathrm{T} 0$ from the values at $\mathrm{T} 2$ (survey variables), and by calculating average improvements (SB). To calculate SB improvements, the SB data at one point in time (e.g. FBM \#3) was subtracted by the SB data at the previous point in time (e.g. FBM \#2), and divided by the previous point in time (e.g. FBM \#2). All resulting differences were then averaged on the individual level.

Tests for statistical significance were two-sided with an alpha of 0.05 , which was corrected using the BenjaminiHochberg procedure [56, 57]. We used $\mathrm{R}$ version 3.4.1 to clean and analyse the data. We used backwards elimination to select the covariates (retention if $p<0.20$ ). As potential covariates we included age, gender, body mass index (BMI), education, work tasks, employment status, and weekly working days. As a result, we controlled for gender (locked in the model) when analysing the intervention effects on SB. For all other models, no covariates were identified.

\section{Results}

\section{Participant Characteristics}

In total, 193 desk workers of companies from different industries (e.g. public service, automotive, education, social service, IT) were willing to participate starting in one of 15 sub-groups (4 sub-groups in intervention group 1, 2 sub-groups in intervention groups 2,3 and 4, and 5 sub-groups in intervention group 5). Of the eligible participants, 43 declined before the kick-offs or did not create an account. Of the 150 participants with an account, 142 started with the baseline week (i.e. wore the VitaBit).
Fig. 2 Number of participants having received specific feedback messages of UPcomplish

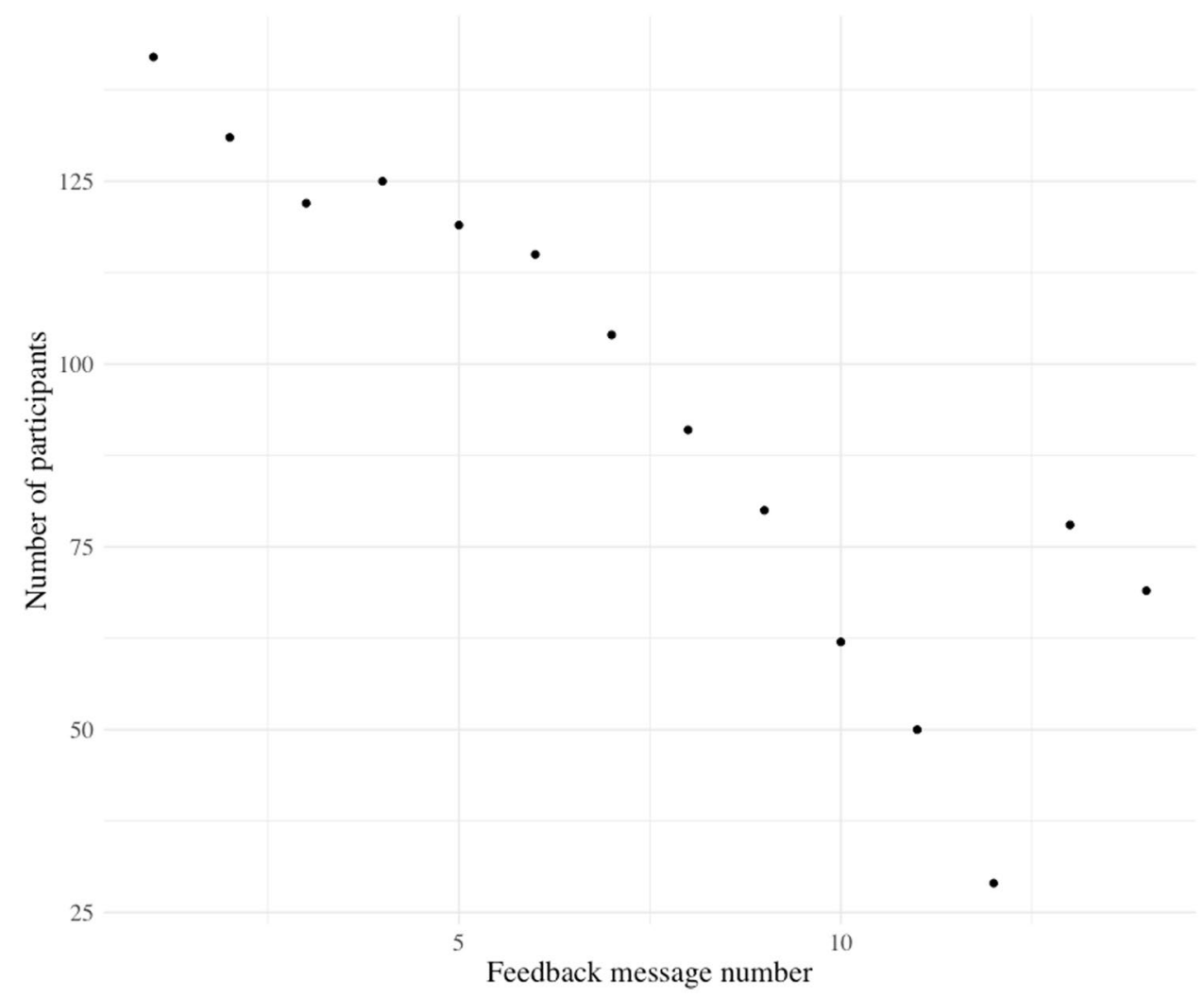


Table 2 Descriptive characteristics of participants at baseline

\begin{tabular}{|c|c|c|c|}
\hline & $\begin{array}{l}\text { Female } \\
n=96\end{array}$ & $\begin{array}{l}\text { Male } \\
n=46\end{array}$ & $\begin{array}{l}\text { Total } \\
n=142\end{array}$ \\
\hline Age (years), median (IQR) & $41.0(20.5)$ & $44.0(19.5)$ & $42.0(21.5)$ \\
\hline \multicolumn{4}{|l|}{ Job-related variables } \\
\hline \multicolumn{4}{|l|}{ Education level, $n(\%)^{\mathrm{a}}$} \\
\hline None & $11(11)$ & $3(7)$ & $14(10)$ \\
\hline Secondary school & $17(18)$ & $10(22)$ & $27(19)$ \\
\hline Professional & $43(45)$ & $23(50)$ & $66(46)$ \\
\hline \multicolumn{4}{|l|}{ Work status, $n(\%)$} \\
\hline Full-time & $65(68)$ & $39(85)$ & $104(73)$ \\
\hline Part-time & $20(21)$ & $1(2)$ & $21(15)$ \\
\hline \multicolumn{4}{|l|}{ Workdays per week, $n(\%)$} \\
\hline 4 workdays & $7(7)$ & $2(4)$ & $9(6)$ \\
\hline 5 workdays & $76(79)$ & $37(80)$ & $113(80)$ \\
\hline 6 workdays & $0(0)$ & $3(7)$ & $3(2)$ \\
\hline \multicolumn{4}{|l|}{ Physical behaviour } \\
\hline Wear time $\left(\min\right.$ day $\left.^{-1}\right)$, mean (SD) & $835.7(102.0)$ & $797.8(115.2)$ & $823.4(107.5)$ \\
\hline Sedentary $\left(\min\right.$ day $\left.^{-1}\right)$, median (IQR) & $504.4(96.5)$ & $522.3(92.7)$ & $510.2(95.3)$ \\
\hline Sedentary compositional geometric mean ${ }^{\mathrm{c}}, \log$-ratio variances standing, walking & $62.3(0.3,0.2)$ & $67.7(0.2,0.2)$ & $64.3(0.3,0.2)$ \\
\hline Standing $\left(\right.$ min day $\left.^{-1}\right)$, median (IQR) & $224.8(129.7)$ & $161.3(73.2)$ & $199.6(102.8)$ \\
\hline Standing compositional geometric mean ${ }^{\mathrm{c}}$, log-ratio variances sitting, walking & $27.2(0.3,0.2)$ & $19.4(0.2,0.1)$ & $24.5(0.3,0.3)$ \\
\hline Activity (min day ${ }^{-1}$ ), median (IQR) & $83.9(45.6)$ & $105.2(37.8)$ & $91.7(45.7)$ \\
\hline Activity compositional geometric mean ${ }^{\mathrm{c}}$, log-ratio variances sitting, standing & $10.5(0.2,0.2)$ & $12.9(0.2,0.1)$ & $11.3(0.2,0.3)$ \\
\hline Program goal achieved (CER), $n(\%)$ & $49(51.0)$ & $14(30.4)$ & $63(44.4)$ \\
\hline \multicolumn{4}{|l|}{ Anthropometrics ${ }^{\mathrm{d}}$} \\
\hline Height $(\mathrm{cm})$ & $168.6(6.9)$ & $180.5(6.7)$ & $172.4(8.8)$ \\
\hline Weight (kg) & $65.0(13.0)$ & $80.0(14.0)$ & $69.0(19.0)$ \\
\hline BMI $\left(\mathrm{kg} / \mathrm{m}^{2}\right)$ & $22.3(5.1)$ & $24.8(3.5)$ & $23.1(4.6)$ \\
\hline Underweight, $n(\%)$ & $5(5)$ & $2(4)$ & $7(5)$ \\
\hline Normal weight, $n(\%)$ & $46(48)$ & $14(30)$ & $60(42)$ \\
\hline Overweight, $n(\%)$ & $16(17)$ & $14(30)$ & $30(21)$ \\
\hline Obese, $n(\%)$ & $4(4)$ & $0(0)$ & $4(3)$ \\
\hline
\end{tabular}

$S D$ standard deviation, IQR interquartile range, $\operatorname{min~}^{-1}$ minutes per day, $\% \mathrm{~d}-1$, proportion of the day, CER control event rate

${ }^{a}$ As indicated during the process of account creation

${ }^{\mathrm{b}}$ Estimates of physical behaviours are estimated via VitaBit accelerometery. Control event rate: Maximum 8 hours sitting, minimum 4 hours standing and walking, $18.8 * 10^{3}$ SSSB on at least $30 \%$ of the days incl. weekend

${ }^{\mathrm{c}}$ The percentage of the day is the estimated proportion of wearing-minutes spent in each activity level

${ }^{\mathrm{d}}$ Underweight defines as BMI $<18.5$, Normal weight 18.5-25, overweight 25-30, obese $>30$

During the intervention, 33 participants dropped out due to technical problems $(n=10)$, because they lost their device $(n=6)$, or due to other reasons, like time constraints $(n=17)$. Of the baseline participants, 109 participants (77\%) stayed in the program until the end as indicated by still having data available and not having indicated to stop the intervention.

The survey was filled out by 129 (91\%), 67 (47\%) and $62(44 \%)$ participants at T0, T1 and T2, respectively. The VitaBit was worn by $109(75 \%)$ and $82(56 \%)$ participants for at least 6 and 9 weeks, respectively. The number of people having received the $\mathrm{n}^{\text {th }}$ FBM decreased from FBM
\#1 ( $n=141$ [99\% of the baseline participants]) to FBM \#12 $(n=29,20 \%)$. FBMs \#13 $(n=78,55 \%)$ and \#14 $(n=69$, $49 \%$ ) were sent to all participants having data at the concerning points in time even if they had message delays. Figure 2 displays the number of participants having been sent FBMs to.

A total of 142 participants (96 females) wore the VitaBit device at baseline (Table 2). Participants had a median age of 42.0 (interquartile range $[\mathrm{IQR}]=21.5$ ) years and a mean BMI of 23.1 (standard deviation $[\mathrm{SD}]=4.6) \mathrm{kg} / \mathrm{m}^{2}$. Of the participants who filled out the survey, the majority worked full-time and worked 5 days per week. At baseline, 63 (44\%) 
Table 3 Multilevel linear models for the effects of different exposures to the UPcomplish intervention on SB

\begin{tabular}{|c|c|c|c|c|c|}
\hline \multirow[t]{2}{*}{ Intervention $^{\mathrm{a}}$} & \multirow[t]{2}{*}{$n^{\mathrm{b}}$} & \multicolumn{2}{|l|}{ SB CoDA } & \multicolumn{2}{|c|}{ Summed squared sitting bouts } \\
\hline & & $\beta(\mathrm{SE})$ & $95 \% \mathrm{CI}$ & $\beta(\mathrm{SE})$ & $95 \% \mathrm{CI}$ \\
\hline 1 & $145(116,29)$ & $-0.01(0.08)$ & $-0.16,0.15$ & $1.25(6.68)$ & $-11.79,14.29$ \\
\hline Intercept & & $-0.03(0.07)$ & $-0.17,0.12$ & $-5.50(6.06)$ & $-17.33,6.34$ \\
\hline 2 & $148(107,41)$ & $-0.01(0.07)$ & $-0.14,0.12$ & $-4.67(5.15)$ & $-14.72,5.38$ \\
\hline Intercept & & $-0.06(0.06)$ & $-0.17,0.06$ & $-8.35(4.52)$ & $-17.18,0.47$ \\
\hline 3 & $149(90,59)$ & $0.01(0.07)$ & $-0.12,0.14$ & $-5.00(5.32)$ & $-15.39,5.38$ \\
\hline Intercept & & $-0.08(0.06)$ & $-0.19,0.02$ & $-8.44(4.40)$ & $-17.03,0.16$ \\
\hline 4 & $111(74,37)$ & $-0.02(0.08)$ & $-0.17,0.13$ & $4.30(6.87)$ & $-9.10,17.70$ \\
\hline Intercept & & $-0.11(0.06)$ & $-0.24,0.02$ & $-11.67(5.82)$ & $-23.02,-0.32$ \\
\hline 5 & $114(20,94)$ & $0.03(0.07)$ & $-0.12,0.18$ & $3.98(6.78)$ & $-9.77,17.07$ \\
\hline Intercept & & $-0.05(0.04)$ & $-0.12,0.03$ & $-5.42(3.73)$ & $-13.20,1.72$ \\
\hline 6 & $126(29,97)$ & $-0.12(0.07)$ & $-0.26,0.03$ & $-9.26(5.80)$ & $-21.07,1.85$ \\
\hline Intercept & & $-0.03(0.05)$ & $-0.12,0.07$ & $-2.45(3.78)$ & $-9.76,5.19$ \\
\hline 7 & $141(40,101)$ & $-0.09(0.06)$ & $-0.21,0.03$ & $-0.34(5.54)$ & $-11.05,9.99$ \\
\hline Intercept & & $-0.03(0.04)$ & $-0.10,0.04$ & $-3.55(3.56)$ & $-9.89,3.23$ \\
\hline 8 & $134(35,99)$ & $-0.04(0.06)$ & $-0.17,0.07$ & $-3.57(5.60)$ & $-14.90,7.15$ \\
\hline Intercept & & $-0.01(0.04)$ & $-0.08,0.06$ & $-1.35(3.87)$ & $-8.79,6.56$ \\
\hline 9 & $119(35,84)$ & $0.01(0.07)$ & $-0.14,0.14$ & $-3.05(6.00)$ & $-15.25,7.77$ \\
\hline Intercept & & $-0.02(0.06)$ & $-0.10,0.08$ & $-2.47(4.65)$ & $-8.50,6.20$ \\
\hline 10 & $96(38,58)$ & $-0.03(0.08)$ & $-0.18,0.11$ & $-0.28(6.53)$ & $-13.23,12.31$ \\
\hline Intercept & & $-0.04(0.06)$ & $-0.16,0.07$ & $-5.41(5.47)$ & $-16.44,5.13$ \\
\hline 11 & $45(29,16)$ & $0.00(0.12)$ & $-0.25,0.24$ & $5.82(8.94)$ & $-13.16,22.94$ \\
\hline Intercept & & $-0.12(0.11)$ & $-0.32,0.09$ & $-8.76(12.50)$ & $-33.83,17.22$ \\
\hline 12 & $18(12,6)$ & $0.22(0.26)$ & $-0.32,0.66$ & $17.24(19.88)$ & $-23.27,52.44$ \\
\hline Intercept & & $-0.05(0.26)$ & $-0.55,0.44$ & $-18.68(19.55)$ & $-55.46,18.19$ \\
\hline 13 & $8(5,3)$ & $0.01(0.16)$ & $-0.33,0.31$ & $-13.49(13.15)$ & $-38.78,11.80$ \\
\hline Intercept & & $0.05(0.14)$ & $-0.22,0.30$ & $0.26(10.40)$ & $-19.73,20.25$ \\
\hline 14 & $80(13,67)$ & $-0.01(0.09)$ & $-0.19,0.16$ & $-1.22(7.30)$ & $-15.42,12.99$ \\
\hline Intercept & & $-0.03(0.04)$ & $-0.11,0.06$ & $-4.81(3.57)$ & $-11.76,2.14$ \\
\hline
\end{tabular}

For the multilevel linear models, the outcome variables were centred around the concerning baseline subgroup means. We adjusted for gender (locked in the model) and clustered by calendar week. Weeks where either no baseline or no respective feedback message was available, were excluded

$C I$ confidence interval, $S E$ standard error

${ }^{*} p<.05 ; * * p<.01 ; * * * p<.001$ (after Benjamini-Hochberg correction)

${ }^{a}$ Feedback message is operationalized as having received this feedback message (and not more or less), which is compared to the baseline measurement of not having received any feedback

${ }^{\mathrm{b}}$ Total number of observations (number of participants having received the concerning feedback message, number of participants at baseline being compared to) participants met the program goal: maximally sitting for $8 \mathrm{~h}$, minimally standing and walking for $4 \mathrm{~h}$, and having a maximum of $18.8 * 10^{3}$ SSSB on at least $30 \%$ of the days (incl. weekend) [27].

\section{Effects of UPcomplish on SB}

Between-subjects, UPcomplish did not result in a significant reduction of SB (Table 3). Within-subjects (Table 6, Appendix B), compared to baseline, participants were significantly more sedentary when they had received FBM \#3 and \#4.

\section{Effects of UPcomplish on QoL}

Neither between-subjects (Table 4) nor within-subjects (Table 7, Appendix C) did the intervention reveal significant effects on QoL.

\section{Effects of UPcomplish on Psychosocial Determinants}

Participants having received FBMs number \#6, \#7, or \#8 reported significantly lower PS compared to baseline (Table 5). Within-subjects (Appendix D, Table 8) compared to baseline, after having received FBMs number \#6, \#7, or 
Table 4 Linear models for the effects of different exposures to the UPcomplish intervention on QoL

\begin{tabular}{|c|c|c|c|c|c|c|c|c|c|}
\hline \multirow[b]{2}{*}{ Intervention $^{\mathrm{a}}$} & \multicolumn{3}{|c|}{ Contextual performance } & \multicolumn{3}{|c|}{ Task performance } & \multicolumn{3}{|c|}{ Perceived stress } \\
\hline & $n^{\mathrm{b}}$ & $\beta(\mathrm{SE})$ & $95 \% \mathrm{CI}$ & $n$ & $\beta(\mathrm{SE})$ & $95 \% \mathrm{CI}$ & $n$ & $\beta(\mathrm{SE})$ & $95 \% \mathrm{CI}$ \\
\hline 1 to 5 & $49(4,45)$ & $-0.26(0.30)$ & $-0.88,0.35$ & $48(4,44)$ & $-0.33(0.27)$ & $-0.87,0.22$ & $48(4,44)$ & $0.58(2.89)$ & $-5.24,6.40$ \\
\hline Intercept & & $-0.03(0.09)$ & $-0.20,0.15$ & & $-0.02(0.08)$ & $-0.18,0.14$ & & $-0.01(0.83)$ & $-1.69,1.67$ \\
\hline 6 to 8 & $101(19,82)$ & $0.23(0.14)$ & $-0.05,0.52$ & $100(19,81)$ & $-0.06(0.15)$ & $-0.36,0.24$ & $100(19,81)$ & $0.40(1.56)$ & $-2.69,3.50$ \\
\hline Intercept & & $-0.02(0.06)$ & $-0.14,0.10$ & & $-0.02(0.07)$ & $-0.15,0.11$ & & $-0.06(0.68)$ & $-1.41,1.29$ \\
\hline 9 to 11 & $100(19,81)$ & $0.09(0.14)$ & $-0.19,0.36$ & $99(19,80)$ & $0.05(0.14)$ & $-0.24,0.33$ & $99(19,80)$ & $-0.77(1.54)$ & $-3.83,2.28$ \\
\hline Intercept & & $-0.02(0.06)$ & $-0.14,0.10$ & & $-0.02(0.06)$ & $-0.15,0.11$ & & $-0.09(0.67)$ & $-1.43,1.25$ \\
\hline 12 to 14 & $79(15,64)$ & $0.05(0.16)$ & $-0.28,0.37$ & $78(15,63)$ & $0.08(0.16)$ & $-0.23,0.40$ & $78(15,63)$ & $-1.49(1.79)$ & $-5.05,2.07$ \\
\hline \multirow[t]{2}{*}{ Intercept } & & $-0.02(0.07)$ & $-0.16,0.12$ & & $-0.02(0.07)$ & $-0.16,0.12$ & & $0.04(0.78)$ & $-1.52,1.60$ \\
\hline & \multicolumn{3}{|c|}{ Perceived pain (inverse) ${ }^{\mathrm{c}}$} & Vitality & & & \multicolumn{3}{|c|}{ Emotional well-being } \\
\hline 1 to 5 & $47(4,43)$ & $4.26(12.09)$ & $\begin{array}{c}-20.09 \\
28.61\end{array}$ & $47(4,43)$ & $-2.04(9.40)$ & $\begin{array}{c}-20.98 \\
16.90\end{array}$ & $47(4,43)$ & $-2.53(6.47)$ & $\begin{array}{c}-15.57 \\
10.51\end{array}$ \\
\hline Intercept & & $-1.19(3.53)$ & $-8.29,5.91$ & & $-0.90(2.74)$ & $-6.43,4.62$ & & $-0.37(1.89)$ & $-4.17,3.44$ \\
\hline 6 to 8 & $99(19,80)$ & $1.57(5.51)$ & $\begin{array}{r}-9.36 \\
12.51\end{array}$ & $99(19,80)$ & $2.88(4.92)$ & $\begin{array}{r}-6.88 \\
12.64\end{array}$ & $99(19,80)$ & $0.07(3.53)$ & $-6.92,7.07$ \\
\hline Intercept & & $-0.35(2.41)$ & $-5.14,4.44$ & & $-0.15(2.15)$ & $-4.42,4.13$ & & $-0.07(1.54)$ & $-3.14,2.99$ \\
\hline 9 to 11 & $97(18,79)$ & $-0.04(6.17)$ & $\begin{array}{c}-12.30 \\
12.21\end{array}$ & $97(18,79)$ & $6.28(5.16)$ & $\begin{array}{r}-3.96 \\
16.51\end{array}$ & $97(18,79)$ & $3.97(3.59)$ & $-3.16,11.10$ \\
\hline Intercept & & $-0.57(2.66)$ & $-5.85,4.71$ & & $-0.48(2.22)$ & $-4.89,3.93$ & & $-0.04(1.55)$ & $-3.11,3.03$ \\
\hline 12 to 14 & $77(15,62)$ & $11.90(6.45)$ & $\begin{array}{r}-0.95 \\
24.76\end{array}$ & $77(15,62)$ & $9.79(5.68)$ & $\begin{array}{r}-1.53 \\
21.11\end{array}$ & $77(15,62)$ & $4.26(4.33)$ & $-4.37,12.90$ \\
\hline Intercept & & $-0.92(2.85)$ & $-6.59,4.76$ & & $-0.60(2.51)$ & $-5.60,4.39$ & & $-0.19(1.91)$ & $-4.00,3.62$ \\
\hline
\end{tabular}

For the linear models, the outcome variables were centred around the baseline sub-group means. Due to singularity, the models were not clustered by calendar weeks. After backwards elimination, no covariates were included. Weeks where either no baseline or no respective feedback message was available, were excluded

$C I$ confidence interval, $S E$ standard error

${ }^{*} p<.05 ; * * p<.01 ; * * * p<.001$ (after Benjamini-Hochberg correction)

${ }^{a}$ Feedback message is operationalized as having received this feedback message (and not more or less), which is compared to the baseline measurement of not having received any feedback

${ }^{\mathrm{b}}$ Total number of observations (number of participants having received the concerning feedback message, number of participants at baseline being compared to)

${ }^{\mathrm{c}}$ Perceived pain is inverted, i.e. higher numbers refers to not having any physical complaints

\#8, they reported significantly lower PS, and after having received FBMs number \#1 to \#5, significantly higher intentions to reduce their $\mathrm{SB}$.

\section{Post Hoc Analyses}

Figure 3 shows the correlations as well as univariate distributions of the variables. Improvement refers to a beneficial development from worse values in the beginning (e.g. more perceived stress, more sitting, less performance) to better values in the end. Firstly, we analysed the correlations of the variables within the clusters (i.e., psychosocial determinants, SB variables, and QoL variables). After Benjamini-Hochberg correction, the improvement of vitality was positively associated with improvement of stress $\left(r=0.57 ; 95 \% \mathrm{CI}=0.25,0.78 ; p<0.01 ; p_{\text {corrected }}=0.02\right)$ and EWB $\left(r=0.64 ; 95 \% \mathrm{CI}=0.35,0.82 ; p=p_{\text {corrected }}<0.001\right)$. Secondly, we studied correlations of improvements in the psychosocial determinants with improvements in SB, which were not found. Thirdly, we examined correlations of improvements in SB with improvements in QoL, which were not found.

\section{Discussion}

This study investigated whether receiving UPcomplish had an effect on SB, QoL and psychosocial determinants as compared to VitaBit only phases. The results suggest that neither on SB nor on QoL does the 12-week intervention have beneficial effects when compared to VitaBit only phases. When having received FBMs \#6, \#7 or \#8, the participants felt less susceptible compared to both their own baseline and others at their baseline, i.e. they agreed less that they should reduce their SB. When having received 5 or fewer FBMs, they indicated higher intentions to reduce and regularly interrupt SB compared to their baseline. None of the improvements in psychosocial determinants was associated 
with improvements in SB, and improvements in SB were not associated with improvements in QoL.

These results are in line with the evaluations of persuasion only interventions that did not reveal SB reductions [34, $58,59]$, notwithstanding any publication bias [60]. Although the relative distribution of the time spent sitting at baseline $(64.3 \%)$ was similar to the distributions that were previously found among office workers [61, 62], already $44 \%$ of the study population met the program goals that had been formulated for the intervention [27]. First, wearing a monitoring device and having received health information during the kick-offs might already have had positive effects [36, 63]. Second, the voluntary participation might have resulted in a selection bias that only participants being interested in a healthy lifestyle participated [64]. This was reflected by positive baseline attitudes towards reducing SB, high baseline QoL, and by the low response rate. Indeed, there seems to be a tendency that interventions with target groups showing more SB at baseline have greater effects on SB compared to target groups with fewer SB [34]. Post hoc analyses within the scope of moderation analyses (in preparation) investigating differences between the participants with more and the ones with less baseline SB are therefore warranted [27, 29].

Several aspects might have impeded the effectiveness of the intervention. Firstly, while environmental changes such as standing desks have been found to be helpful $[18,34,36]$, the individual employee possesses limited possibilities to reduce sitting at work due to, for example, time constraints and the ways that work is structured (e.g. lengthy meetings at round-tables). Additionally, SB has become a habitual process because it is linked to diverse contexts and activities, such as work, and standing at work is perceived as uncomfortable [65-67]. Unsurprisingly, therefore, that others only found significant SB reductions on weekends [20, 26]. SB might be less of a reasoned behaviour and more determined by environmental, societal, or habitual factors. This is also reflected in the fact that none of the improvements in psychosocial determinants was associated with improvements in SB. Secondly, due to the high baseline QoL values and the selectivity of the sample [64], short-term effects of this intervention such as reductions of back-pain or an increase of vitality might not have been as dominant in order to serve as additional motivators [68], which was also reflected by the poor correlations between improvements in SB and QoL. Thirdly, the FBMs of this intervention merely focused on workplace $\mathrm{SB}$. Since this constitutes the majority of the daily life, we had expected an overall reduction of SB. Nevertheless, while it might have had a reducing effect only on workplace sitting, leisure time habits (after working hours and weekends) might have mitigated the effects, which was already found in other SB interventions focusing on workplace sitting [24, 25]. Lastly, there seems to be a tendency that the intervention reduced SB after 6 to 8 FBMs, but the perceived need to sit

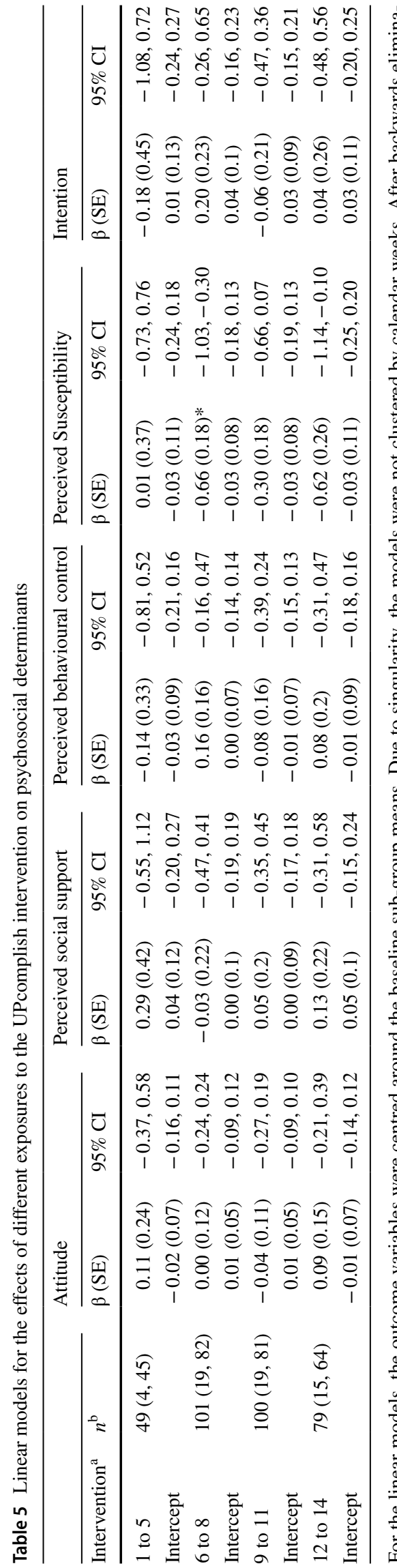




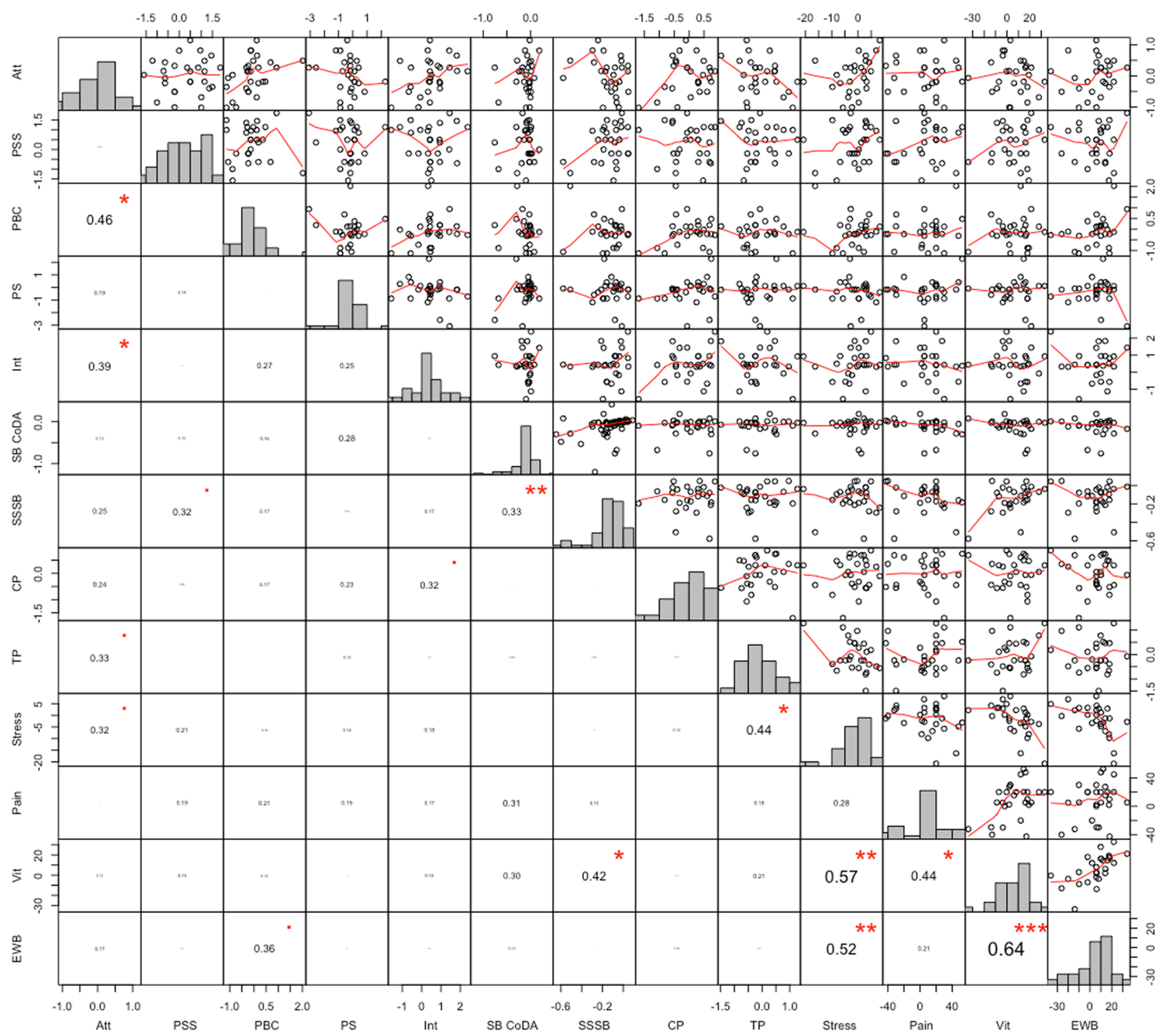

Fig. 3 Pearson correlations and plots illustrating the linear and smoothed associations, respectively, between improvements in determinants (T2-T0), SB (week to week) and QoL (T2-T0). Abbreviations: Att, attitude; PSS, perceived social support; PBC, perceived behavioural control; PS, perceived susceptibility; Int, intention; SB

less (i.e. PS) also drops around this moment. This might have been a reason why higher intentions to reduce SB after the first few FBMs were not stable. To keep participants motivated, they were provided with a lot of positive feedback on their progress. Additional to positive feedback, it might, hence, be important to still remind participants of their initial goals and general SB recommendations. Blom et al. evaluated two personal counselling interventions and only found an increase in controlled and autonomous motivation after the physical activity intervention and not after the SB intervention [67]. It might be helpful to have another personal meeting in the middle of the intervention, to keep sending
CoDA, SB proportion; SSSB, summed squared sitting bouts; CP, contextual performance; TP, task performance; Stress, perceived stress; Pain, not having any pain; Vit, perceived vitality; EWB, emotional well-being

two FBMs per week, or to adapt the FBMs themselves, also with a greater focus on healthy alternative behaviours and reminding of SB recommendations.

\section{Strengths and Limitations}

The current study examined the effects that a workplace SB intervention has on overall daily sitting. While many interventions only analyse the effects on workplace sitting, this study focuses on the target group and their entire daily life providing better external validity and a valid predictor for participants' general health. Furthermore, the analyses 
respected the compositional and inter-dependent nature of physical behaviours, and included a novel, yet intuitive, operationalization of prolonged sitting. Additionally, the drop-out rates were smaller compared to other workplace physical activity and SB interventions, and they were mostly due to technical problems rather than a loss of motivation [69]. This is an indicator of the acceptability and, thereby, the potential of the UPcomplish intervention. Lastly, the stepped-wedge design revealed more data points and reliability per participant, and allowed for high external validity since data were collected throughout $75 \%$ of the year.

The study also has some limitations. Firstly, we assume a recruitment bias among participants which might have resulted in a group of participants being dominated by females, being healthier and more motivated than the average office employee. Nonetheless, baseline SB proportions of participants are comparable to what was found in previous studies. Secondly, we included participants of diverse workplace sites and with potential underlying health problems (e.g. cardiac problems), which might not be comparable in terms of SB and the potential to reduce it. Nonetheless to increase internal and external validity, we centred all outcome variables around baseline company means, and included multiple company industries, education levels, team and company sizes. Additionally, we not only conducted between-subjects but also within-subjects statistical analyses which increased statistical power and generalizability of the results. Similarly, since all FBMs of the intervention were tailored, the effects might not be fully comparable across subjects. However, all FBMs were drafted based on the change objectives and the parameters of effectiveness of the UPcomplish intervention. This ensured that each participant received FBMs relevant to them, which is one of the requirements for the methods to be effective in behaviour change. Lastly, the kick-off session prior to the UPcomplish intervention could be considered as an additional intervention. Yet, previous research did not find effects of providing generic information on SB and its consequences and this study investigated the effects of providing tailored feedback.

This study provides an essential addition to the literature on SB. Although UPcomplish was structurally developed using evidence from the literature and from theory, it was neither effective in improving SB nor QoL. In the middle of the intervention, participants perceived to be less susceptible to being sedentary. Firstly, we conclude that a workplace SB intervention might need to focus more on structural changes of the workplace environment. Secondly, workplace sitting might not only be influenced by the psychosocial determinants that were chosen for this intervention, but also by other psychosocial determinants or by different underlying beliefs. Lastly, the sample seemed to be selective in such a way that the participants were likely more motivated, less sedentary and had higher QoL compared to the average office worker. In an additional study, potential moderators of the effectiveness of UPcomplish will be investigated to explore whether UPcomplish was effective for certain subgroups, such as people being more sedentary.

Supplementary Information The online version contains supplementary material available at https://doi.org/10.1007/s12529-022-10054-0.

Acknowledgements We would like to thank VitaBit software for providing access to the measurement toolkits and the data. Furthermore, thank you very much to all participating office workers. We thank Tugce Varol for providing some final edits to this paper.

Author Contribution Nathalie M. Berninger: conceptualization, funding acquisition, visualization, project administration, software, methodology, writing — original draft, investigation. Guy Plasqui: supervision, visualization, methodology, writing — review and editing. Rik Crutzen: supervision, visualization, software, methodology, writing — review and editing. Robert A. C. Ruiter: supervision, funding acquisition, writing-review and editing. Gerjo Kok: supervision, writing — review and editing. Gill A. ten Hoor: supervision, funding acquisition, methodology, writingreview and editing.

Availability of Data and Material The cleaned raw data and additional material is fully disclosed in the supplementary materials (https://osf. io/qzp9m/?view_only=30ada8d6fc0e4ac19a1610b8901f9f96).

\section{Declarations}

Ethics Approval, Consent to Participate, Consent for Publication This study and its consent procedure was approved by the Ethics Review Committee of the Faculty of Psychology and Neuroscience, Maastricht University, the Netherlands [ERCPN-188_11_02_2018].

Conflict of Interest Nathalie M. Berninger was employed by VitaBit to develop and evaluate UPcomplish. However, apart from providing the devices and access to the software and the raw data for the current study, VitaBit had no role in the design of the study; in the analyses, or interpretation of data; in the writing of the manuscript, and in the decision to publish the results.

Open Access This article is licensed under a Creative Commons Attribution 4.0 International License, which permits use, sharing, adaptation, distribution and reproduction in any medium or format, as long as you give appropriate credit to the original author(s) and the source, provide a link to the Creative Commons licence, and indicate if changes were made. The images or other third party material in this article are included in the article's Creative Commons licence, unless indicated otherwise in a credit line to the material. If material is not included in the article's Creative Commons licence and your intended use is not permitted by statutory regulation or exceeds the permitted use, you will need to obtain permission directly from the copyright holder. To view a copy of this licence, visit http://creativecommons.org/licenses/by/4.0/.

\section{References}

1. Carter S, et al. Sedentary behavior and cardiovascular disease risk: mediating mechanisms. Exerc Sport Sci Rev. 2017;45(2):80-6.

2. Patterson R, et al. Sedentary behaviour and risk of all-cause, cardiovascular and cancer mortality, and incident type 2 diabetes: 
a systematic review and dose response meta-analysis. Springer. 2018.

3. Magnon V, Vallet GT, Auxiette C. Sedentary behavior at work and cognitive functioning: A systematic review. Front Public Health. 2018;6:239.

4. Tremblay MS, et al. Sedentary behavior research network (SBRN)-terminology consensus project process and outcome. Int J Behav Nutr Phys Act. 2017;14(1):75.

5. Healy GN, et al. Breaks in sedentary time beneficial associations with metabolic risk. Diabetes Care. 2008;31(4):661-6.

6. Bankoski A, et al. Sedentary activity associated with metabolic syndrome independent of physical activity. Diabetes Care. 2011;34(2):497-503.

7. Duvivier BMFM, et al. Breaking sitting with light activities vs structured exercise: a randomised crossover study demonstrating benefits for glycaemic control and insulin sensitivity in type 2 diabetes. Diabetologia. 2017;60(3):490-8.

8. Voss MW, et al. Revenge of the "sit" II: does lifestyle impact neuronal and cognitive health through distinct mechanisms associated with sedentary behavior and physical activity? Ment Health Phys Act. 2014;7(1):9-24.

9. Wennberg $\mathrm{P}$, et al. Acute effects of breaking up prolonged sitting on fatigue and cognition: a pilot study. BMJ Open. 2016;6(2):e009630-e009630.

10. Hendriksen IJM, et al. Longitudinal relationship between sitting time on a working day and vitality, work performance, presenteeism, and sickness absence. J Occup Environ Med. 2016;58(8):784-9.

11. Hamilton MT, et al. Too little exercise and too much sitting: inactivity physiology and the need for new recommendations on sedentary behavior. Curr Cardiovasc Risk Rep. 2008;2(4):292-8.

12. Alzahrani $\mathrm{H}$, et al. (320) The association between sedentary behavior and low back pain: a systematic review and metaanalysis of longitudinal studies. J Pain. 2019;20(4):S55.

13. Chen S-M, et al. Sedentary lifestyle as a risk factor for low back pain: a systematic review. Int Arch Occup Environ Health. 2009;82(7):797-806.

14. Coenen P, et al. Associations of screen work with neck and upper extremity symptoms: a systematic review with metaanalysis. Occup Environ Med. 2019;76(7):502-9.

15. Rebar AL, et al. Differences in health-related quality of life between three clusters of physical activity, sitting time, depression, anxiety, and stress. BMC Public Health. 2014;14(1):1088.

16. Faulkner G, Biddle S. Standing on top of the world: is sedentary behaviour associated with mental health? Ment Health Phys Act. 2013;6(1):1-2.

17. Prince SA, et al. Device-measured physical activity, sedentary behaviour and cardiometabolic health and fitness across occupational groups: A systematic review and meta-analysis. Int J Behav Nutr Phys Act. 2019;16(1):30.

18. Pereira MA, et al. Efficacy of the 'Stand and Move at Work' multicomponent workplace intervention to reduce sedentary time and improve cardiometabolic risk: a group randomized clinical trial. Int J Behav Nutr Phys Act. 2020;17(1):1-11.

19. Shimo K, et al. Effects of a 12-week workplace counseling program on physical activity and low back pain: a pilot randomized controlled study. J Back Musculoskeletal Rehab. 2020:1-8.

20. Arrogi A, et al. Short-and long-term effectiveness of a threemonth individualized need-supportive physical activity counseling intervention at the workplace. BMC Public Health. 2017;17(1):1-20.

21. Schoeppe $S$, et al. Efficacy of interventions that use apps to improve diet, physical activity and sedentary behaviour: a systematic review. Int J Behav Nutr Phys Act. 2016;13(1):127.
22. Broekhuizen $\mathrm{K}$, et al. A systematic review of randomized controlled trials on the effectiveness of computer-tailored physical activity and dietary behavior promotion programs: an update. Ann Behav Med. 2012;44(2):259-86.

23. Kelders SM, et al. Persuasive system design does matter: a systematic review of adherence to web-based interventions. I Med Internet Res. 2012;14(6):e152.

24. De Cocker K, et al. The effectiveness of a web-based computertailored intervention on workplace sitting: a randomized controlled trial. J Med Internet Res 2016;18(5):e96.

25. Morris AS, et al. Rise and recharge: effects on activity outcomes of an e-health smartphone intervention to reduce office workers' sitting time. Int J Environ Res Public Health. 2020;17(24):9300.

26. Bort-Roig J, et al. An mHealth workplace-based "Sit Less, Move More" program: impact on employees' sedentary and physical activity patterns at work and away from work. Int J Environ Res Public Health. 2020;17(23):8844.

27. Berninger NM, et al. Sedentary work in desk-dominated environments: a data-driven intervention using intervention mapping. J Med Internet Res Formative Res. 2020;4(7):e14951.

28. O'Cathain A, et al. Taxonomy of approaches to developing interventions to improve health: a systematic methods overview. Pilot Feasibility Stud. 2019;5(1):41.

29. Bartholomew Eldredge LK, et al. Planning health promotion programs: an intervention mapping approach. John Wiley \& Sons. 2016.

30. Coffeng JK, et al. Effectiveness of a worksite social \& physical environment intervention on need for recovery, physical activity and relaxation; results of a randomized controlled trial. PloS One. 2014;9(12):e114860.

31. Kwak L, et al. Changes in skinfold thickness and waist circumference after 12 and 24 months resulting from the NHF-NRG In Balance-project. Int J Behav Nutr Phys Act. 2010;7(1):26.

32. McEachan RR, et al. Evidence, theory and context: using intervention mapping to develop a worksite physical activity intervention. BMC Public Health. 2008;8(1):326-38.

33. Fishbein M, Ajzen I. Predicting and changing behavior: the reasoned action approach. Taylor \& Francis. 2011.

34. Stephenson A, et al. Using computer, mobile and wearable technology enhanced interventions to reduce sedentary behaviour: a systematic review and meta-analysis. Int J Behav Nutr Phys Act. 2017;14(1):105.

35. Kok G, et al. A taxonomy of behaviour change methods: an intervention mapping approach. Health Psychol Rev. 2016;10(3):297-312.

36. Gardner B, et al. How to reduce sitting time? A review of behaviour change strategies used in sedentary behaviour reduction interventions among adults. Health Psychol Rev. 2016;10(1):89-112.

37. Berninger NM, ten Hoor GA, Plasqui G. Validation of the vitabit sit-stand tracker: detecting sitting, standing, and activity patterns. Sensors. 2018;18(3):877.

38. Hemming $\mathrm{K}$, et al. The stepped wedge cluster randomised trial: rationale, design, analysis, and reporting. British Med J. 2015;350:h391.

39. Hemming K, et al. Reporting of stepped wedge cluster randomised trials: extension of the CONSORT 2010 statement with explanation and elaboration. British Med J. 2018;363:k1614.

40. Klepac Pogrmilovic B, et al. National physical activity and sedentary behaviour policies in 76 countries: availability, comprehensiveness, implementation, and effectiveness. Int J Behav Nutr Phys Act. 2020;17(1):1-13.

41. Atkin AJ, et al. Methods of measurement in epidemiology: sedentary behaviour. Int J Epidemiol. 2012;41(5):1460-71.

42. Brislin RW. Back-translation for cross-cultural research. J Cross Cult Psychol. 1970;1(3):185-216. 
43. Crutzen R, Peters G-JY. Scale quality: alpha is an inadequate estimate and factor-analytic evidence is needed first of all. Health Psychol Rev. 2017;11(3):242-7.

44. Revelle W, Zinbarg RE. Coefficients alpha, beta, omega, and the glb: Comments on Sijtsma. Psychometrika. 2009;74(1):145.

45. De Cocker K, et al. Theory-driven, web-based, computer-tailored advice to reduce and interrupt sitting at work: development, feasibility and acceptability testing among employees. BMC Public Health. 2015;15(1):1.

46. Koopmans L, et al. Improving the individual work performance questionnaire using rasch analysis. J Appl Meas. 2014;15(2):160-75.

47. Cohen S, Kamarck T, Mermelstein R. Perceived stress scale. Measuring Stress: A Guide Health Soc Sci. 1994;235-283.

48. Klein EM, et al. The German version of the Perceived Stress Scale-psychometric characteristics in a representative German community sample. BMC Psychiatry. 2016;16(1):159.

49. Ware JE Jr. SF-36 health survey update. Spine. 2000;25(24):3130-9.

50. Kim J, Park HA. Development of a health information technology acceptance model using consumers' health behavior intention. J Med Internet Res. 2012;14(5).

51. Champion VL, Skinner CS. The health belief model, in Health behavior and health education: Theory, research, and practice, $\mathrm{K}$. Glanz, B.K. Rimer, and K. Viswanath, Editors. 2008;45-65.

52. Busschaert $\mathrm{C}$, et al. Reliability and validity of three questionnaires measuring context-specific sedentary behaviour and associated correlates in adolescents, adults and older adults. Int J Behav Nutr Phys Act. 2015;12:117.

53. Chastin SF, et al. Combined effects of time spent in physical activity, sedentary behaviors and sleep on obesity and cardio-metabolic health markers: a novel compositional data analysis approach. PloS One. 2015;10(10):e0139984.

54. Toftager M, et al. Accelerometer data reduction in adolescents: effects on sample retention and bias. Int J Behav Nutr Phys Act. 2013;10(1):140.

55. Mahalanobis PC. On the generalized distance in statistics. Nat Inst Sci India. 1936.

56. Benjamini Y, Yekutieli D. The control of the false discovery rate in multiple testing under dependency. Ann Stat. 2001;1165-1188.

57. Benjamini Y, Hochberg Y. Controlling the false discovery rate: a practical and powerful approach to multiple testing. J Roy Stat Soc: Ser B (Methodol). 1995;57(1):289-300.
58. Direito A, et al. mHealth technologies to influence physical activity and sedentary behaviors: Behavior change techniques, systematic review and meta-analysis of randomized controlled trials. Ann Behav Med. 2017;51(2):226-39.

59. Patel AK, Banga C, Chandrasekaran B. Effect of an educationbased workplace intervention (move in office with education) on sedentary behaviour and well-being in desk-based workers: a cluster randomized controlled trial. Int J Occup Safety Ergonom. 2021;1-9.

60. Reed JL, et al. Impact of workplace physical activity interventions on physical activity and cardiometabolic health among workingage women: a systematic review and meta-analysis. Circ Cardiovasc Qual Outcomes. 2017;10(2):e003516.

61. Ryan CG, et al. Sitting patterns at work: objective measurement of adherence to current recommendations. Ergonomics. 2011;54(6):531-8.

62. Clemes SA, O'Connell SE, Edwardson CL. Office workers' objectively measured sedentary behavior and physical activity during and outside working hours. J Occup Environ Med. 2014;56(3):298-303.

63. Barwais FA, Cuddihy TF, Tomson LM. Physical activity, sedentary behavior and total wellness changes among sedentary adults: a 4-week randomized controlled trial. Health Qual Life Outcomes. 2013;11(1):183.

64. Waters LA, et al. Who participates in physical activity intervention trials? J Phys Act Health. 2011;8(1):85-103.

65. Conroy DE, et al. Sedentary behavior as a daily process regulated by habits and intentions. Health Psychol. 2013;32(11):1149.

66. Maher JP, Conroy DE. A dual-process model of older adults' sedentary behavior. Health Psychol. 2016;35(3):262-72.

67. Blom V, et al. The effects on self-efficacy, motivation and perceived barriers of an intervention targeting physical activity and sedentary behaviours in office workers: a cluster randomized control trial. BMC Public Health. 2021;21(1):1-9.

68. Segar ML, et al. From a vital sign to vitality: selling exercise so patients want to buy it. Curr Sports Med Rep. 2016;15(4):276-81.

69. Cajita MI, et al. Feasible but not yet efficacious: a scoping review of wearable activity monitors in interventions targeting physical activity, sedentary behavior, and sleep. Curr Epidemiol Rep. 2020;7(1):25-38.

Publisher's Note Springer Nature remains neutral with regard to jurisdictional claims in published maps and institutional affiliations. 